\title{
ACTUALIZACIÓN DE CIRUGÍA BARIÁTRICA Y CIRUGÍA METABÓLICA
}

\author{
UPDATE OF BARIATRIC \\ AND METABOLIC SURGERY
}

Dras. María Graciela R. Álvarez, Susana Fuentes, Juliana Mociulsky, Victoria Salinas, María Yuma, Susana Gutt, Marianela Ackerman, Paula Lipzig, Dr. Frank Espinoza Morales

Coordinadora: Dra. Carla Musso

Revisores: Dres. José E. Costa Gil, León E. Litwak, Isaac R. Sinay

\section{RESUMEN}

La obesidad aumenta el riesgo de desarrollar o agravar múltiples afecciones particularmente la diabetes tipo 2, una enfermedad crónica de difícil control cuyo tratamiento con distintos fármacos y aún con insulina, frecuentemente no alcanza los objetivos terapéuticos ni reduce el riesgo cardiovascular. Cuando se asocia a obesidad severa, su terapéutica se torna más compleja. Se analiza la utilización de cirugía bariátrica y metabólica en pacientes con obesidad severa y diabetes, se revisan las técnicas quirúrgicas, las hipótesis sobre su mecanismo de acción, la selección de pacientes y los resultados. La cirugía bariátrica ha probado ser efectiva en el tratamiento de la obesidad severa y de la DM2 en pacientes con IMC $>35 \mathrm{~kg} / \mathrm{m}^{2}$. Es una cirugía de aceptable tolerancia en personas preparadas, de bajo riesgo y con complicaciones subsanables y manejables en la actualidad. La cirugía bariátrica dispone de suficiente respaldo científico. En cambio, en las evidencias disponibles de la cirugía metabólica en pacientes con IMC $<35 \mathrm{~kg} / \mathrm{m}^{2}$ y más aún con IMC $<30 \mathrm{~kg} / \mathrm{m}^{2}$ existen resultados promisorios en el corto y mediano plazo, pero no se dispone aún de evidencia suficiente que avale su indicación. Se requieren más investigaciones para establecer la eficacia y seguridad de estas técnicas quirúrgicas en el largo plazo.

Palabras clave: diabetes tipo 2, obesidad mórbida, cirugía bariátrica, cirugía metabólica.

Revista de la Sociedad Argentina de Diabetes 2013; Vol. 4769 - 80

Comité de Obesidad y Diabetes. Sociedad Argentina de Diabetes Correspondencia: Carla Musso

E-mail: mussocar@hotmail.com

Paraguay 1307, piso $8^{\circ}$ ofic. 74 (C1057AAU), CABA, Argentina

Tel./Fax: 4816-2838 /4815-1379

\section{ABSTRACT}

Obesity increases the risk of developing or worsening many disorders particularly type 2 diabetes, a difficult-to-control chronic disease, treated with different drugs even with insulin, frequently without reaching therapeutic goals neither reducing cardiovascular risk. Therapy becomes more complex when type 2 diabetes is associated with severe obesity. The use of bariatric and metabolic surgery in patients with severe obesity and diabetes is assessed, and the different surgical techniques, assumptions about its mechanism of action, selection of patients and results are also reviewed. Bariatric surgery has proven to be effective in the treatment of severe obesity and type 2 diabetes in patients with $B M I>35 \mathrm{~kg} / \mathrm{m}^{2}$. This type of surgery has shown to be acceptable and well tolerated in selected and low risk patients capable of treatment Bariatric surgery has sufficient scientific evidence in these patients. However, there is no strong evidence of metabolic surgery in patients with $B M l<35 \mathrm{~kg} / \mathrm{m}^{2}$ and even with $B M l<30 \mathrm{~kg} / \mathrm{m}^{2}$. Preliminary data shows promising results in the short and intermediate term, but there is no sufficient evidence available supporting its indication in these patients. More research is needed to establish the efficacy and safety of these surgical techniques in the long term.

Key words: Type 2 diabetes, morbid obesity, bariatric surgery, metabolic surgery.

Revista de la Sociedad Argentina de Diabetes 2013; Vol. 4769 - 80

\section{INTRODUCCIÓN}

La obesidad aumenta el riesgo de desarrollar o agravar múltiples afecciones. En la actualidad se define de acuerdo al índice de masa corporal (IMC) (Tabla 1) pero hay que considerar además del peso, la cuantía de la masa grasa, su distribución e importantes aspectos funcionales del tejido adiposo. Se asocia con diabetes tipo 2 (DM2) y otras comorbilidades: hipertensión arterial, dislipidemia, síndrome de apnea obstructiva del sueño, enfermedad cardiovascular, varios tipos de cáncer y trastornos de la fertilidad, entre otras. La obesidad mórbida (IMC $>40 \mathrm{~kg} / \mathrm{m}^{2}$ ) afecta la calidad y disminuye la expectativa de vida?. 


\begin{tabular}{|l|c|l|}
\hline Peso corporal & $\begin{array}{c}\text { Índice de } \\
\text { Masa Corporal }\end{array}$ & Riesgo \\
\hline Bajo peso & $<18,5$ & \\
\hline Normal & $18,5-24,9$ & Saludable \\
\hline Sobrepeso & $25,0-29,9$ & $\begin{array}{l}\text { Aumentado: } \\
\text { leve }\end{array}$ \\
\hline $\begin{array}{l}\text { Obesidad } \\
\text { grado I }\end{array}$ & $30,0-34,9$ & $\begin{array}{l}\text { Aumentado: } \\
\text { moderado }\end{array}$ \\
\hline $\begin{array}{l}\text { Obesidad } \\
\text { grado II }\end{array}$ & $35,0-39,9$ & $\begin{array}{l}\text { Aumentado: } \\
\text { severo }\end{array}$ \\
\hline $\begin{array}{l}\text { Obesidad } \\
\text { grado III }\end{array}$ & $40,0-55,9$ & Muy severo \\
\hline $\begin{array}{l}\text { Súper } \\
\text { obesidad }\end{array}$ & 50,0 ó $>$ & Muy severo \\
\hline $\begin{array}{l}\text { Súper súper } \\
\text { obesidad }\end{array}$ & 60,0 ó $>$ & Muy severo \\
\hline
\end{tabular}

Tabla 1: Criterios diagnósticos y clasificación del estado nutricional de acuerdo al IMC, según la OMS ${ }^{11}$.

Una elevada proporción de personas con DM2 tiene sobrepeso/obesidad y aunque no todos los obesos tienen DM, su frecuencia aumenta con la severidad de la obesidad y llega a más del $30 \%$ entre obesos mórbidos. Según datos de la Encuesta Nacional de Factores de Riesgo de 2009, la prevalencia de diabetes fue del 9,6\% en la población general, se incrementó a 19,9\% en los pacientes con obesidad grado 1 y a $31,9 \%$ en los pacientes con obesidad grado 3. Resulta preocupante que en la población pediátrica obesa, la tolerancia a la glucosa alterada es, en la actualidad, el trastorno metabólico más frecuente y se detecta DM2 en aproximadamente el $2,5 \%$ de los niños y adolescentes obesos ${ }^{2}$.

La DM2 es una enfermedad crónica de difícil control cuyo tratamiento con distintos fármacos y aún con insulina, frecuentemente no alcanza los objetivos terapéuticos, ni reduce el riesgo cardiovascular así como tampoco las complicaciones crónicas de la enfermedad. Cuando se asocia a obesidad severa, su terapéutica se torna más compleja dado que algunos medicamentos y la intensificación del tratamiento suelen acentuar y acelerar el aumento del peso. Asimismo, los pacientes con DM tienen mayor dificultad para descender de peso ${ }^{3,4}$.

Una reducción modesta del peso (5-7\%) mediante intervenciones en el estilo de vida, evita o retarda la aparición de DM2 en sujetos con riesgo y mejora el control glucémico en los pacientes con $\mathrm{DM}^{5-8}$. Sin embargo, estos cambios son difíciles de sostener en el largo plazo y es frecuente una nueva ganancia de peso. En los pacientes con obesidad severa, este tipo de tratamiento fracasa y la disminución del $10 \%$ del peso corporal parece insuficiente para lograr beneficios en la salud. Para controlar los factores de riesgo cardiovascular a 10 años, se requeriría un descenso del 10 al $30 \%$ del peso sostenido en el tiempo ${ }^{9,10}$.

En el presente trabajo de revisión se analizan aspectos que vinculan a la DM2 y a la obesidad, en especial del tipo mórbida. Se discuten las estrategias quirúrgicas actuales para resolver la difícil situación que viven los pacientes y el equipo de salud frente a la DM y la obesidad severa.

\section{Historia de la cirugía de la obesidad}

En los últimos 50 años se han considerado múltiples técnicas quirúrgicas para el tratamiento de la obesidad mórbida: de tipo restrictivas, malabsortivas, combinaciones de ambas, estimulación eléctrica, balón intragástrico y dispositivos gastro-intestinales innovadores. En 1966 Mason realizó la primera serie de cirugías de bypass gástrico en-Y-Roux ${ }^{12,13}$.

A comienzos de la década de 1980 se realizaron técnicas puramente restrictivas con la colocación de anillos o bandas gástricas fijas para reducir la capacidad gástrica. Estas técnicas han caído en desuso.

En 1991 el National Institutes of Health $(\mathrm{NIH})$ de Estados Unidos, en reunión de consenso sobre "Cirugía Gastrointestinal de la Obesidad Severa" consideró a la cirugía bariátrica como una técnica útil y efectiva para el tratamiento de la obesidad mórbida y estableció las guías para la selección adecuada de pacientes, que se mantiene hasta la actualidad. Reconoció a la obesidad mórbida como una enfermedad severa que acorta la vida y produce o agrava otras patologías y que responde en forma pobre o nula a los tratamientos convencionales; asimismo se aceptó por consenso que la cirugía bariátrica es el tratamiento de elección para esta enfermedad, con un abordaje interdisciplinario de cirujanos, clínicos, nutricionistas y psicólogos ${ }^{14}$.

La actualización de las guías NIH en 1996 afirmó que la cirugía es el único tratamiento efectivo de la obesidad severa ${ }^{15}$. Diferentes sociedades científicas americanas y europeas coincidieron con las guías del $\mathrm{NIH}$, con diferencias menores en los criterios de selección de los pacientes ${ }^{16,17}$.

En el año 2007 se reunió el "Consenso Cumbre en Cirugía en Diabetes" donde 50 expertos (29 de ellos no eran cirujanos) acordaron las recomendaciones de la cirugía metabólica en las que se reco- 
noció por primera vez un concepto innovador: la cirugía como tratamiento específico para la DM2 en pacientes cuidadosamente seleccionados. Aconsejaron la cirugía en pacientes con DM2 e IMC $\geq 35$ $\mathrm{kg} / \mathrm{m}^{2}$ (nivel de consenso A: $90-100 \%$ de los participantes estuvo de acuerdo), en coincidencia con las guías del NIH 1991 y de la ADA 2009 y la consideraron una alternativa terapéutica en pacientes con IMC entre 30 y $35 \mathrm{~kg} / \mathrm{m}^{2}$ con mal control de la DM2 bajo tratamiento con cambios en el estilo de vida y fármacos (nivel de consenso $B$, acuerdo 7889\%). El bypass gástrico en-Y-Roux sería la opción quirúrgica apropiada para estos pacientes (nivel de consenso C: acuerdo 67-88\%). Precisaron que en pacientes con IMC $<30 \mathrm{~kg} / \mathrm{m}^{2}$, la cirugía sólo debe considerarse dentro de protocolos de investigación debidamente aprobados por los comités de ética ${ }^{18}$.

Los cuatro procedimientos que se utilizan en la actualidad inicialmente se clasificaron según sus mecanismos de acción restrictivos y/o malabsortivos, aunque debería reconsiderarse la nomenclatura de acuerdo a los actuales conocimientos sobre los péptidos intestinales y las actividades de los ejes entero insular y entero encefálico. Dos técnicas son estrictamente restrictivas: la banda gástrica ajustable (Figura 1) y la manga gástrica (sleeve, Figura 2), una mixta (restrictiva-malabsortiva), el bypass gástrico en-Y-Roux (que se considera "el patrón de oro" del tratamiento de la obesidad y DM2, Figura 3) y la restante es fundamentalmente malabsortiva, la derivación biliopancreática, con una variante: el switch duodenal ${ }^{19}$.

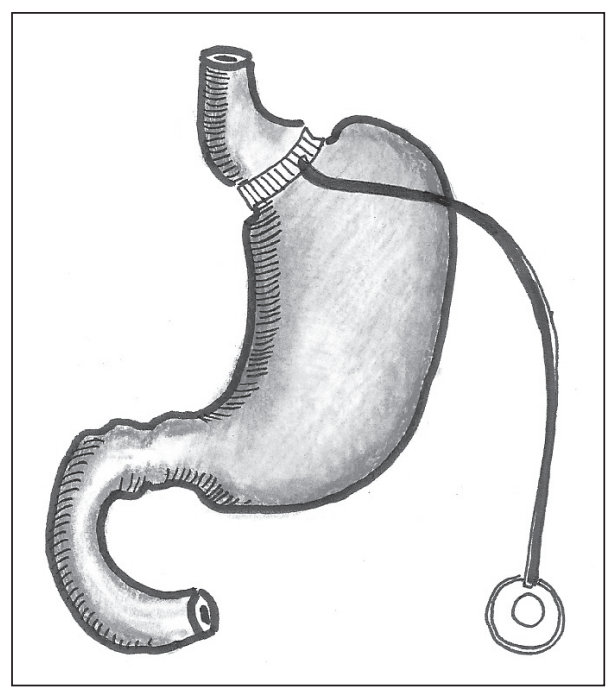

Figura 1: Banda gástrica ajustable con puerto subcutáneo (M.G. Álvarez).

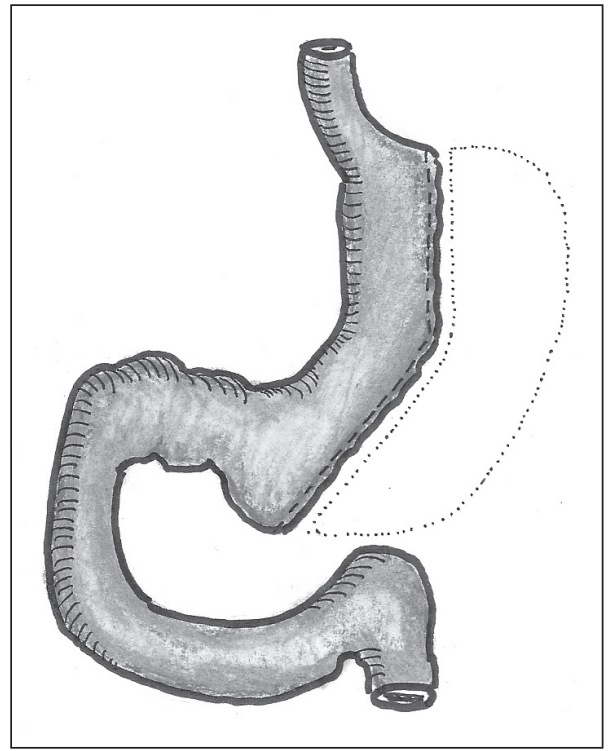

Figura 2: Gastrectomía vertical en manga. En línea punteada, el cuerpo gástrico que se reseca en la cirugía (M.G. Álvarez).

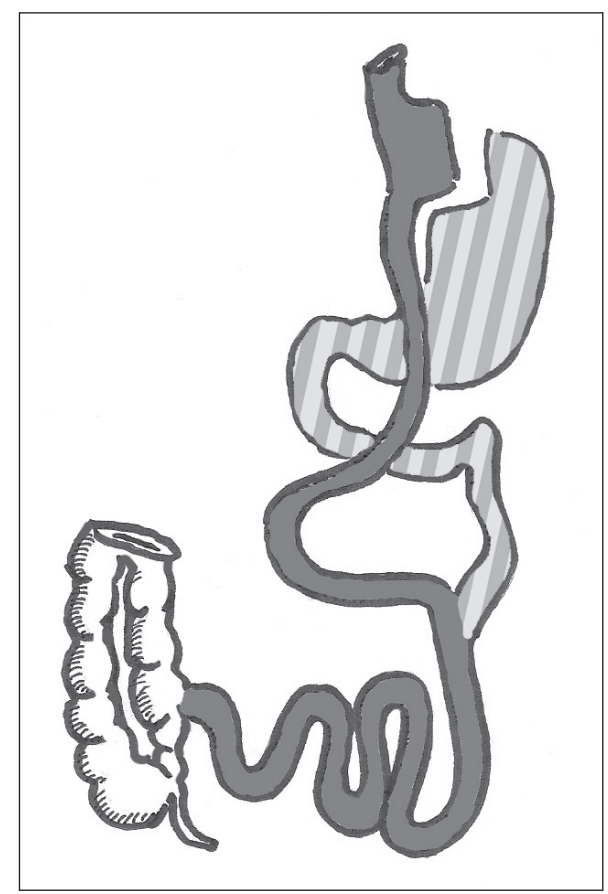

Figura 3: Bypass gástrico en-Y-de-Roux. A rayas el asa biliar, en color gris osuro el pouch gástrico se continúa con el yeyuno: el asa alimentaria y en color blanco el asa común donde se reúnen los alimentos con las enzimas digestivas y se produce la absorción (M.G. Álvarez).

\section{Conceptos sobre cirugía bariátrica y cirugía metabólica}

La cirugía bariátrica (del griego baros = peso) es el tratamiento quirúrgico de la obesidad mórbida 
que se define como IMC > a $40 \mathrm{~kg} / \mathrm{m}^{2}$ ó IMC > a 35 $\mathrm{kg} / \mathrm{m}^{2}$ acompañado de alguna comorbilidad. Es una intervención sobre el tubo digestivo que logra un descenso substancial del peso y su mantenimiento en el largo plazo. También es un recurso eficiente para la prevención y el control de la $\mathrm{DM} 2^{20}$.

Se denomina cirugía metabólica a la intervención por medio de la cual se redirecciona el tránsito de los alimentos en el tubo digestivo para mejorar la DM2 por mecanismos independientes a la disminución del peso, en pacientes con un IMC < a $35 \mathrm{~kg} / \mathrm{m}^{2}$. Algunos autores denominan Diabetología Intervencionista al abordaje terapéutico de la DM2 con cirugía 21 .

La DM2 es una enfermedad progresiva que recibe tratamiento médico con la meta terapéutica de evitar o retrasar la aparición de complicaciones crónicas. En cambio, la cirugía de la diabetes propone otro objetivo: la remisión completa de la afección, con glucemias $<100 \mathrm{mg} / \mathrm{dL}$ y $\mathrm{HbA} 1 \mathrm{c}<6 \%$, sin necesidad de farmacoterapia para el control de la glucemia y por consiguiente, con impacto en dichas complicaciones. Se considera remisión completa duradera a la que se mantiene por más de 5 años y remisión parcial a la hiperglucemia por debajo del rango diabético, con glucemia de ayunas $110-125 \mathrm{mg} / \mathrm{dL}$ y hemoglobina glicosilada $<6,5 \%{ }^{14}$.

En el postoperatorio inmediato de la cirugía bariátrica, los pacientes con DM2 mejoran los valores de glucemia y disminuyen el requerimiento de medicamentos con un efecto que se sostiene a través del tiempo, como se observó en un estudio de 1.160 pacientes sometidos a bypass gástrico. De los 240 sujetos con DM2, el 30\% obtuvo el alta hospitalaria a las 48 hs., sin requerimiento de medicación (incluidos aquellos que se trataban con insulina) $)^{22}$.

Se han descripto otros efectos beneficiosos sobre las comorbilidades de la obesidad: resuelve o mejora el síndrome de apnea obstructiva del sueño ${ }^{23}$, la dislipidemia ${ }^{24}$, la hipertensión arterial ${ }^{25}$, los trastornos mecánicos como la osteoartritis ${ }^{26}$, el reflujo gastro-esofágico, la incontinencia urinaria y fundamentalmente restablece la calidad de vida y el estado psicosocial de los individuos. $\mathrm{Ha}$ demostrado que disminuye el riesgo cardiovascu$\operatorname{lar}^{27,28}$ y aumenta la expectativa de vida de los pacientes obesos operados en comparación con los que recibieron tratamiento médico 29-30.

La DM2 es la comorbilidad de la obesidad que más sensiblemente se corrige con esta interven- ción quirúrgica y mejora en aproximadamente el $80 \%$ de los $\operatorname{casos}^{31-35}$.

En un metaanálisis en el que se revisaron 136 estudios con más de 22.000 casos, se observó la remisión de la DM2 en el $84 \%$ de los pacientes operados de bypass gástrico y en el $98 \%$ de las cirugías de derivación biliopancreática ${ }^{36}$ (Tabla 2).

\begin{tabular}{|l|c|c|c|}
\hline Parámetro & $\begin{array}{c}\text { Banda } \\
\text { gástrica } \\
\text { ajustable }\end{array}$ & $\begin{array}{c}\text { Bypass } \\
\text { gástrico } \\
\text { en Y-Roux }\end{array}$ & $\begin{array}{c}\text { Derivación } \\
\text { biliopancreática }\end{array}$ \\
\hline $\begin{array}{l}\% \text { exceso } \\
\text { de peso } \\
\text { perdido }\end{array}$ & $48-68 \%$ & $84 \%$ & $72 \%$ \\
\hline $\begin{array}{l}\text { Remisión } \\
\text { de la DM2 }\end{array}$ & $48-72 \%$ & $84 \%$ & $98 \%$ \\
\hline $\begin{array}{l}\text { Reducción } \\
\text { de la HTA }\end{array}$ & $28-73 \%$ & $75 \%$ & $81 \%$ \\
\hline $\begin{array}{l}\text { Mejoría } \\
\text { de la disli- } \\
\text { pidemia }\end{array}$ & $71-81 \%$ & $94 \%$ & $100 \%$ \\
\hline $\begin{array}{l}\text { Disminu- } \\
\text { ción de la } \\
\text { mortalidad }\end{array}$ & $0,1 \%$ & $0,5 \%$ & $1,1 \%$ \\
\hline
\end{tabular}

Tabla 2: Resultados del metaanálisis para determinar el impacto de la cirugía bariátrica sobre el peso corporal y comorbilidades, en este caso, diabetes tipo 2.

\section{Factores predictivos de la respuesta de la DM2 a la cirugía}

Los mejores resultados en la resolución quirúrgica de la DM2 se observaron en los pacientes con pocos años de evolución de la misma, que no requerían insulina, tenían niveles de péptido-C conservados y buen control glucémico preoperatorio $^{12}$. En la medida que aumentó la antigüedad de la DM2, disminuyeron las probabilidades de éxito terapéutico. Así, con menos de 5 años de evolución se obtuvo el $95 \%$ de remisión, entre 5 y 10 años, un $75 \%$ y por encima de ese tiempo se observó un $56 \%$ de buenos resultados en el tratamiento de la DM2. La mayor duración de la DM2 se acompaña con mayor deterioro irreversible de la célula $\beta$.

La técnica quirúrgica también influye sobre la eficacia del tratamiento y la remisión. En las intervenciones restrictivas, la mejoría depende principalmente del descenso de peso, mientras que en las técnicas malabsortivas se producirían por mecanismos independientes del peso ${ }^{37,38}$. 
La cirugía también previene nuevos casos de diabetes. En el estudio SOS que siguió la evolución de los pacientes a los 10 y 15 años, la incidencia de DM2 disminuyó un 96, 84 y 78\% respectivamente, en comparación con el grupo control, con lo que se alcanzó aproximadamente el doble de resultados favorables comparado con los logrados en programas de cambios intensivos en el estilo de vida ${ }^{39}$. La cirugía tiene mayor efecto preventivo en aquellos sujetos con glucemia en ayunas alterada que con glucemia normal. Por el contrario, el IMC no predice el efecto preventivo de la cirugía bariátrica sobre la $\mathrm{DM}^{40,41}$. Se debe destacar que aún están en proceso de evaluación las indicaciones de la cirugía metabólica.

A pesar de los beneficios descriptos, sólo un $1 \%$ de los pacientes en condiciones para ser tratado con cirugía accede a este tratamiento. Son varias y diversas las razones que generan esta situación e incluyen: la discriminación contra las personas con obesidad y sus tratamientos, las barreras por parte de la comunidad médica que considera a la DM2 y a la obesidad de preferente tratamiento médico-farmacológico, la falta de información y formación sobre las opciones quirúrgicas, las dificultades de accesibilidad, además de los costos y temores por su invasividad, efectividad y seguridad en la que se destacan los riesgos potenciales, principalmente a largo plazo ${ }^{42,43}$.

Se enfatizan los beneficios independientes de la pérdida del peso en relación a la remisión o mejoría de la DM2:

1. El restablecimiento metabólico se observa con gran rapidez luego del bypass gástrico en sólo días o semanas, aún antes de un descenso significativo de peso.

2. Es más intenso luego de la cirugía de bypass gástrico en $Y$ de Roux en comparación con una pérdida de peso equivalente alcanzado con otras técnicas quirúrgicas o con tratamiento médico.

Asimismo es importante destacar que se ha observado también una mejoría de la tolerancia a la glucosa luego de técnicas experimentales que no utilizaron mecanismos restrictivos ni malabsortivos y por consiguiente no conllevan una disminución de la ingesta, tales como el bypass duodenoyeyunal, la manga endoluminal (Endobarrier R) o la interposición ileal.

\section{Criterios actuales de inclusión y exclusión de pacientes para cirugía bariátrica}

\section{Criterios de inclusión}

1. $I M C>40 \mathrm{~kg} / \mathrm{m}^{2}$.

2. $\mathrm{IMC}>35 \mathrm{~kg} / \mathrm{m}^{2}$ con comorbilidades asociadas.

3. Edad de 18 a 65 años.

4. Obesidad de por lo menos 5 años de evolución con fracaso de tratamientos conservadores debidamente supervisados.

5. Compromiso del paciente a adherir a controles postquirúrgicos, concurrir para el seguimiento del equipo tratante, cuidados alimentarios, toma de suplementos vitamínicos, entre otros aspectos.

Se encuentra en revisión la utilidad del IMC como criterio para la indicación de cirugía bariátrica ya que no define en el paciente individual, los riesgos y beneficios. Es, además, un predictor imperfecto del compromiso metabólico. Por el otro lado, el IMC no pronostica apropiadamente los efectos de la cirugía sobre el descenso de peso, la remisión de la DM2, la prevención de cáncer o enfermedad cardiovascular. Tendrían más importancia otras variables metabólicas, como la glucemia y la insulinemia en ayunas ${ }^{4-46}$.

\section{Criterios de exclusión}

1. Enfermedades endócrinas reversibles.

2. Abuso de alcohol o drogas en el momento actual.

3. Enfermedad psiquiátrica severa y no controlada.

4. Falta de comprensión del paciente de los beneficios y riesgos, y de la necesidad de cambios en el estilo de vida.

5. Existencia de expectativas irreales.

6. Enfermedad de base con corta expectativa de vida.

Deben evaluarse individualmente los casos de pacientes adolescentes o añosos con valoración de riesgos y beneficios de cada caso particular.

Las indicaciones y contraindicaciones se modifican periódicamente de manera dinámica, por diversos motivos:

1. Aumento marcado de la prevalencia de obesidad mórbida.

2. Disponibilidad de técnicas bariátricas más seguras con baja morbimortalidad.

3. Auge de la cirugía laparoscópica mínimamente invasiva y de avances tecnológicos.

4. Mayor experiencia y abordaje en equipos multidisciplinarios.

5. Mejor conocimiento sobre la cirugía bariátrica 
en poblaciones especiales tales como adolescentes y adultos mayores.

La posibilidad de remisión completa de la DM2 (¿curación?) disminuye cuando se indica tardíamente este tipo de cirugía. Así lo muestran los datos de intervención temprana sobre costo/efectividad, mejoría de las comorbilidades (dislipidemia, hipertensión arterial, apnea obstructiva del sueño, etc.) y aumento de la expectativa de vida de los pacientes operados ${ }^{47}$.

\section{Criterios de éxito de la cirugía bariátrica}

1. Disminución de $50 \%$ del exceso de peso (peso inicial-peso actual) / (peso inicial-peso ideal) $x$ 100, a los 5 años.

2. Logro de $I M C<35 \mathrm{~kg} / \mathrm{m}^{2}$.

3. Mejora o resolución de las comorbilidades.

4. Restablecimiento de la calidad de vida.

\section{Consideraciones}

En el año 2011 la Federación Internacional de Diabetes comunicó su posición sobre la cirugía bariátrica para el tratamiento de pacientes con obesidad y DM2. En sus aspectos sobresalientes expresó:

1. Existen opciones eficaces para mejorar la DM en pacientes con obesidad severa, además de los enfoques de tratamientos médicos y conductuales, a través de diversos tipos de cirugía en el tracto gastrointestinal desarrollados originalmente para tratar la obesidad mórbida ("cirugía bariátrica"), con los que frecuentemente se normaliza la glucemia, disminuye o evita la necesidad de medicamentos y conforma un recurso rentable de tratamiento.

2. Constituye una terapéutica adecuada para pacientes con obesidad y DM2 que no alcanzan los objetivos con tratamiento médico, en especial en presencia de otras comorbilidades.

3. Se debe considerar en los algoritmos de tratamiento de la DM2.

4. Se acepta en pacientes con $I M C>40 \mathrm{~kg} / \mathrm{m}^{2}$ y se considera una opción en pacientes con IMC $>35 \mathrm{~kg} / \mathrm{m}^{2}$ y en casos seleccionados con IMC entre 30 y $35 \mathrm{~kg} / \mathrm{m}^{2}$.

5. Los prejuicios sociales sobre la obesidad severa, que existen aún dentro del sistema de atención de salud, no deben actuar como una barrera para la opción a este tratamiento clínicamente eficaz y costo-efectivo.

6. La morbilidad y la mortalidad asociadas a la cirugía bariátrica son generalmente bajas y simi- lares a las de procedimientos bien aceptados, como la vesícula biliar o cirugía de litiasis biliar.

7. En pacientes severamente obesos con DM tiene beneficios para la salud que incluye la reducción de todas las causas de mortalidad.

8. Se requiere de investigación continua y se enfatiza la necesidad de identificar pacientes con distintos fenotipos para definir cuál sería la técnica bariátrica más adecuada según edad, género y duración de la DM2. Se recomienda realizar dosaje de péptido $C$ y anticuerpos como el anti GAD en pacientes seleccionados ${ }^{48-49}$.

\section{Conceptos acerca de criterios de inclusión, exclusión y de éxito de la cirugía metabólica}

Estudios de observación recientes demuestran que los procedimientos de cirugía bariátrica disminuyen la incidencia de diabetes tipo 2 y causan una mejoría marcada o resolución en muchos pacientes con enfermedad preexistente. Si bien algunos médicos consideran que la cirugía bariátrica es un recurso extremo, los datos sugieren que los procedimientos quirúrgicos producen beneficios importantes para la salud de los pacientes con DM2 e IMC >35 kg/m², y plantean si no se debe recurrir antes a ellos en el desarrollo de la enfermedad o frente a un exceso de peso menor y específicamente para el tratamiento de la DM en oposición al de la obesidad.

En este momento son imprescindibles estudios prospectivos aleatorizados que evalúen si el tratamiento médico o el quirúrgico son óptimos para el tratamiento de la DM2 en pacientes con obesidad moderada (IMC $<35 \mathrm{~kg} / \mathrm{m}^{2}$ ). La investigación se debe orientar a identificar las características de los pacientes que tienen buenos resultados y mejores evoluciones que los demás con el fin de personalizar las recomendaciones terapéuticas cuando sea posible ${ }^{50}$.

Los criterios de inclusión que deben considerarse al seleccionar pacientes candidatos a cirugía metabólica incluyen: pacientes con diagnóstico de DM2, con IMC 30-35 kg/m² e inadecuado control de su enfermedad mediante cambios en el estilo de vida y tratamiento farmacológico, con dosaje de péptido $C>1 \mathrm{pcg} / \mathrm{dL}$ en ayunas y marcadores de autoinmunidad negativos. Asimismo se puede utilizar el péptico $C$ posprandial (en la prueba oral de tolerancia a la glucosa o la prueba de tolerancia a comida mixta) en la que se considera que $1 \mathrm{U}$ de incremento en el índice se asocia con un 30\% de 
aumento en la probabilidad de mejorar el control de la glucemia a los 2 años.

Los predictores de remisión de la enfermedad se asociaron fuertemente con la antigüedad y la severidad de la $\mathrm{DM} 2^{51}$.

\section{Hipótesis sobre mecanismos posibles de mejoría de la DM2 por cirugía bariátrica}

Existen controversias acerca de cuáles son los factores que conducen a la mejoría metabólica luego de las intervenciones quirúrgicas bariátricas en la DM2. Se han fundamentado en la recuperación de la sensibilidad hepática a la glucosa por la restricción energética, aunque en la actualidad no se limitan sólo a este mecanismo.

Las primeras propuestas se centraron en procesos de restricción y de malabsorción. Sin embargo, ha crecido la aceptación sobre los cambios en las hormonas enterales y los eventos neuronales que se producen por los cambios anatómicos pos-BGYR. Éstos involucran una comunicación directa con el sistema nervioso central para regular la conducta alimentaria y el equilibrio energético y los cambios en la fisiología del hígado, del tejido adiposo, de los músculos y del páncreas que modifican directa e indirectamente la glucemia y la secreción y acción de la insulina. Se han planteado múltiples hipótesis acerca de los mecanismos de estos efectos.

\section{Hipótesis 1: del intestino distal}

Se basa en los cambios demostrados en las incretinas y otras respuestas neuroendocrinas a partir de un aporte de nutrientes más directo hacia el intestino distal52. La importancia del aporte temprano de nutrientes a la porción distal del intestino delgado tiene respaldo en los experimentos de transposición ileal, en los cuales una porción del íleon se traslada a una ubicación por encima de la unión duodenoyeyunal, lo que causa menor ingesta de comida, descenso de peso sin malabsorción de grasas y se asocia con aumento de GLP-1, péptido YY, preproglucagon y mejoría metabólica ${ }^{53}$.

\section{Hipótesis 2: del intestino proximal}

Se postula que el desvío del flujo de nutrientes que evita la porción proximal del intestino delgado elimina el estímulo para la producción de una señal diabetogénica (por la posible presencia de hormonas anti-incretinas que aún no se han identificado), como se comprobó en una serie de experimentos quirúrgicos en la que se demostró la mejoría en las glucemias de ratas con el bypass duodeno-yeyunal, lo cual no sucedió con ratas sometidas a una operación quirúrgica simulada o con tratamiento dietético con ratas diabéticas no obesas Goto-Kakizaki. Las mejorías en la glucemia no persistieron cuando se restableció el tránsito gastroyeyunal de nutrientes ${ }^{54,55}$. También se han observado mejorías en la glucemia sin descenso de peso en humanos con la colocación de una endoprótesis por vía endoscópica (Endobarrier) que excluye al duodeno del contacto con los alimentos y con intervenciones de bypass duodeno-yeyunal. Tal vez el BGYR es tan efectivo para disminuir el peso y la hiperglucemia porque reúne los múltiples mecanismos mencionados.

\section{Hipótesis 3: efecto de las hormonas intestinales}

El estudio de las hormonas entéricas luego de las distintas intervenciones quirúrgicas bariátricas y de su relación con los cambios metabólicos y el descenso de peso sostenido, constituye un campo por explorar. Una de las hormonas entéricas más estudiada es la grelina, que se sintetiza en el estómago. Las concentraciones aumentan antes de las comidas y estimulan el apetito. Hay lípidos específicos de la dieta que actúan como sustratos para la acilación de la grelina, lo que modifica su actividad y con ello brinda un mecanismo de comunicación entre la disponibilidad de nutrientes y el estado metabólico ${ }^{56}$. Con el descenso de peso inducido por la dieta, aumentan las concentraciones de grelina, situación que incrementa el apetito y favorece la recuperación del peso perdido. En contraste, luego del BGYR disminuyen los niveles de grelina, se reduce la actividad de búsqueda de comida y favorece el mantenimiento del descenso de peso. Al mismo tiempo, aumentan los péptidos producidos luego de las comidas a partir de las células $L$ del íleon, como el GLP-1, el péptido YY y la oxintomodulina, probablemente debido a un aporte temprano de nutrientes al íleon y a mecanismos neuronales ${ }^{52}$. Todos estos péptidos se asocian con la saciedad, lo que ayudaría a mantener el descenso de peso. Estos cambios no se observan con la banda gástrica o el descenso de peso inducido por dieta. Algunos de las variaciones en las hormonas entéricas, como el aumento de los niveles del GLP-1, pueden favorecer la regeneración o el mantenimiento de la masa o la función de los islotes pancreáticos. En estudios de experimentación se mostró que después del 
BGYR aumenta la expresión de la homeocaja 1 pancreático-duodenal, un factor de transcripción temprano y esencial en el linaje de la célula $\beta$ y la captación de bromodesoxiuridina a las células $\beta$ (un análogo sintético de la timidina que se incorpora al ADN de la célula cuando durante el proceso de división), lo que promueve efectos positivos sobre el islote ${ }^{57}$. Sin embargo, aún despiertan controversias los cambios en la masa o la función del islote pancreático luego del BGYR.

\section{Otras hipótesis}

- Gluconeogénesis intestinal. Se ha observado también que el BGYR mejora la sensibilidad hepática y reduce la gluconeogénesis hepática sin afectar la sensibilidad periférica a la insulina. Se considera que el BGYR promueve la gluconeogénesis intestinal, ya que el aumento de glucosa en la vena porta estimularía el sensor hepatoportal de glucosa vía GLUT2 y disminuiría la producción hepática de glucosa. La pérdida de la respuesta gluconeogenética se asocia a la ausencia de efecto antidiabético luego de la cirugía.

Estos hallazgos sugieren que la gluconeogénesis intestinal contribuye al ajuste de la homeostasis glucémica post BGYR ${ }^{58}$.

- Sales biliares. Se estima que los cambios en el flujo de bilis por las modificaciones anatómicas explicarían algunos de los efectos metabólicos de la cirugía bariátrica. Se ha demostrado que luego de BGYR junto al aumento directo de incretinas también se elevan ácidos biliares plasmáticos.

Los ácidos biliares estimularían las células $L$ del íleon responsables de la producción de GLP1. Al desviar la bilis al íleon distal, aumentaría la respuesta de GLP1 con mejora de la glucemia.

Las sales biliares también estarían involucradas en la reducción de la liberación hepática de glucosa y en los efectos metabólicos de la microbiota que cambia luego del BGYR ${ }^{59}$.

\section{Alimentación en la cirugía bariátrica}

Todos los pacientes sometidos a cirugía bariátrica, cualquiera sea la técnica que se utilice, deben recibir soporte para evitar un fallo en el cierre de las suturas durante los primeros días, como para impedir o resolver déficits nutricionales $u$ otras complicaciones médicas y para prevenir reganancia de peso. Las pautas alimentarias se tie- nen que adaptar a la nueva anatomía y fisiología del tracto gastrointestinal.

Las técnicas quirúrgicas actuales reducen la ingesta calórica total y también hay intolerancia selectiva a ciertos alimentos que limitan su consumo.

\section{Estrategias nutricionales previas a la cirugía de la obesidad}

El objetivo principal es lograr un descenso de peso del $10 \%$ del exceso, que permita:

1. Reducir las posibles complicaciones médicoquirúrgicas asociadas a la obesidad.

2. Mejor recuperación postquirúrgica.

\section{Valor calórico total}

Se disminuyen de 600 a 1.000 calorías/día de la ingesta habitual para llegar a planes alimentarios de 1.200 a 1.600 calorías, trabajando sobre el cambio de hábitos alimentarios con el propósito de corregir alteraciones nutricionales y aliviar o mejorar patologías gastrointestinales y metabólicas. En los días previos a la cirugía (1 a 2 semanas) se utilizan dietas de muy bajo valor calórico para acelerar la disminución del volumen hepático.

El tiempo de seguimiento previo a la cirugía es variable y depende de la situación clínico-psicológica, el compromiso y adherencia del paciente a los cambios propuestos. En el Consenso Argentino de Nutrición en Cirugía Bariátrica se consideró que este período debe ser no menor a tres meses, con consultas periódicas como mínimo 1 vez al mes en el contexto multidisciplinario.

\section{Estrategias nutricionales posteriores a la cirugía de la obesidad. Recomendaciones generales}

1. No se debe beber con las comidas; hacerlo una hora antes ó 45 minutos después. Los líquidos se tienen que tomar lentamente, a temperatura ambiente y evitar los fríos o calientes extremos (primera etapa). Incorporar líquidos con la comida puede favorecer la aparición de náuseas y vómitos.

2. Masticar muy bien los alimentos, emplear entre 20 y 30 minutos en cada comida para impedir dolor y vómitos.

3. No tomar mate, gaseosas, alcohol ni bebidas azucaradas para evitar el Síndrome de Dumping.

4. Comer tranquilamente y sin leer, mirar televisión o usar la computadora.

5. Dejar de comer antes de sentir sensación de plenitud. 
6. Cumplir estrictamente con el plan previamente pautado ajustándose a la progresión prescripta.

\section{Recomendaciones específicas}

- Intervenciones restrictivas: importa disminuir el volumen de la ingesta porque el reservorio gástrico residual es de 25 a $50 \mathrm{ml}$ de promedio. Se deben ingerir pequeños volúmenes en reiteradas oportunidades para cubrir las necesidades calórico-proteicas.

- Intervenciones mixtas y malabsortivas: la alimentación posterior a la cirugía consta de cuatro etapas cuya duración depende de la tolerancia de cada paciente.

- Primera etapa - 1a: líquidos claros sin azúcar ni gas a temperatura ambiente de 24 a 48 hs. post-cirugía. El volumen será de 1.500-2.500 cc diario.

- Primera etapa - 1 b: dieta líquida proteica (desde el tercero al décimo día del postquirúrgico). El paciente debe tomar al menos $2.000 \mathrm{a}$ $2.500 \mathrm{ml}$ por día con 60 a $80 \mathrm{~g} /$ día de proteínas (se debe indicar suplementación proteica para lograr el objetivo proteico).

- Segunda etapa: dieta tipo papillas o puré (a partir de los 10 días hasta el mes) se incluyen carnes blancas hervidas y trituradas.

- Tercera etapa: dieta blanda (desde el primero al segundo mes). Se incorporan carnes rojas hasta llegar a la dieta libre saludable donde se puede comer en forma variada proteínas magras, frutas y verduras.

- Cuarta etapa: desde los 45 a 60 días según tolerancia del paciente, plan alimentario saludable, con aproximadamente 1.200 calorías y con $1 \mathrm{~g} / \mathrm{kg} / \mathrm{día}$ de peso ideal en proteínas.

Debido a que la ingesta es escasa, al comienzo no se puede garantizar un adecuado aporte de micronutrientes, por ello deben utilizarse complejos con vitaminas y minerales, al margen de los suplementos específicos para cada caso como hierro, calcio, vitaminas $A, B 12$ y D, zinc y ácido fólico. Estos micronutrientes se deberán determinar periódicamente.

\section{Consideraciones especiales}

- Náuseas y vómitos: son las complicaciones post-cirugía más frecuentes y precoces. Pueden aparecer como consecuencia de comer demasiado rápido o ingerir volúmenes que superen la capacidad del reservorio gástrico, por masticación insuficiente, mezclar sólidos y líquidos o por la incorporación de un nuevo alimento. Si son repentinos, en una buena progresión alimentaria pueden indicar una complicación quirúrgica: estenosis de la neoboca.

- Diarrea: más común en los casos de derivación biliopancreática que en las cirugías restrictivas y malabsortivas.

- Estreñimiento: puede deberse a la baja ingesta de fibra y grasa, a una inadecuada hidratación o a trastornos de la motilidad intestinal.

- Síndrome de Dumping: la aparición de sudoración, náuseas y/o vómitos, malestar general, frialdad, sensación de apetito, puede ser consecuencia de la ingesta de alimentos ricos en hidratos de carbono simples.

- Intolerancias alimentarias: son idiosincráticos a cada persona. Las más comunes son a las carnes rojas, seguidas de los vegetales crudos, arroz, pan blanco y cereales integrales. Se deben enseñar técnicas culinarias para mejorar la tolerancia a las comidas.

- Dolor abdominal: se puede producir por ingesta excesiva, mala masticación, comer rápido o tomar bebidas con gas o a temperaturas extremas, particularmente en los primeros meses.

- Deshidratación: puede aparecer por vómitos, diarrea o escasa ingesta de líquidos. Se debe insistir en cumplir con los volúmenes pautados, en forma lenta, fraccionada y alejada de las comidas.

\section{El rol de los grupos terapéuticos en pacientes obesos en plan de cirugía bariátrica}

Para evitar recaídas en el proceso de adaptación a los cambios es necesario un enfoque multidisciplinario para el tratamiento. Este enfoque para el tratamiento del paciente obeso en plan de cirugía bariátrica debe considerar:

1. Evaluación psicológica.

2. Control nutricional.

3. Seguimiento médico.

4. Grupo terapéutico.

5. La inclusión del paciente en grupos terapéuticos $^{60,61}$ puede mejorar la adherencia al tratamiento y brinda a los miembros la posibilidad de desarrollar capacidades o potencialidades y a su vez compartir un problema común. En este contexto se define grupo como un conjunto de personas que tiene intereses y problemas comunes y se reúne para resolverlos ${ }^{62}$. 
Se han descripto diversos beneficios del trabajo grupal en salud ${ }^{63,64}$. A diferencia de las presentaciones informativas, el grupo genera espíritu de pertenencia y tiene continuidad en el tiempo. Al ser el hombre un ser social, se une a otros hombres para solucionar sus problemas.

Asimismo se han enumerado varios factores beneficiosos asociados al grupo, los cuales son variados y actúan interdependientemente en todo tipo de actividad grupa|65. Los más importantes son:

1. Esperanza: al compartir con otros la experiencia positiva, aumentan las expectativas de recuperación.

2. Universalidad: al compartir el problema, se toma contacto con la realidad y el participante se da cuenta que no está solo, que no sólo a él le sucede lo que ocurre.

3. Información: el conocimiento lleva a la acción. Para el cambio real se necesita algún tipo de aprendizaje cognitivo.

4. Altruismo: recibir a través de dar, es un proceso recíproco.

5. Reproducción del grupo familiar: se repiten modos de relación familiar, conflictos, afectos, rivalidades, competencias.

6. Socialización: se facilita el conocimiento personal, no sólo por percibirse uno mismo sino a través del contacto con los demás.

7. Cohesión: es la experiencia de sentirse aceptado y ser capaz de compartir ideas y sentimientos con los demás sin temor a críticas.

8. Factores existenciales: aprender y sentir que tiene la responsabilidad final de la vida que lleva, además de la guía y del soporte de los demás.

Los grupos de apoyo para pacientes bariátricos ofrecen la posibilidad de interacción entre pacientes ya operados y aquellos que aún están en una etapa informativa o en plan quirúrgico. La oportunidad de discutir sobre los distintos aspectos relacionados a la intervención permite también una mejor preparación y conocimiento de los cuidados postquirúrgicos ${ }^{66}$.

\section{DISCUSIÓN}

La cirugía bariátrica ha probado ser efectiva en el tratamiento de la obesidad severa y de la DM2 en pacientes con $\mathrm{IMC}>35 \mathrm{~kg} / \mathrm{m}^{2}$. Es una cirugía de aceptable tolerancia en personas preparadas, de bajo riesgo y con complicaciones subsanables y manejables en la actualidad.

Se debe distinguir la consideración de la ciru- gía bariátrica, que dispone de suficiente respaldo científico, de las evidencias disponibles de la cirugía metabólica. Para los pacientes con IMC $<35$ $\mathrm{kg} / \mathrm{m}^{2}$ y más aún, con $\mathrm{IMC}<30 \mathrm{~kg} / \mathrm{m}^{2}$ existen resultados promisorios en el corto y mediano plazo, pero no se dispone aún de evidencia suficiente que avale su indicación. Se requieren más investigaciones para establecer la eficacia y seguridad de estas técnicas quirúrgicas en el largo plazo.

\section{REFERENCIAS}

1. www.who.int/mediacentre/factsheets/fs311. Última revisión $1 / 6 / 2013$.

2. Zimmet P, Shaw J and Albert KG. Preventing Type 2 diabetes and the dysmetabolic syndrome in the real world: a realistic view. Diabet Med. 2003; 20:693-702.

3. Khan MA, St Peter JV, Breen GA, Hartley GG, Vessey JT. Diabetes disease stage predicts weight loss outcomes with longterm appetite suppressants. Obes Res. 2000; 8:43-48.

4. Zimmet P, Shaw J, Alberti KG. Preventing type 2 diabetes and the dysmetabolic syndrome in the real world: a realistic view. Diabet Med. 2003; 20:693-702.

5. Pan X, Li G, Hu Y, Wang J, Yang W, An Z Hu ZX, Lin J, Xiao JZ, Cao HB, Liu PA, Jiang XG, Jiang YY, Wang JP, Zheng $H$, Zhang $\mathrm{H}$, Bennett $\mathrm{PH}$, Howard BV. Effects of diet and exercise in preventing NIDDM in people with impaired glucose tolerance: the DaOing IGT Diabetes Study. Diabetes Care 1997; 20: 537-544.

6. Tuomilehto J, Lindstrom J, Eriksson J, Valle T, Hamalainen $\mathrm{H}$, llanne-Parikka $P$ Keinänen-Kiukaanniemi S, Laakso M, Louheranta A, Rastas M, Salminen V, Uusitupa M; Finnish Diabetes Prevention Study Group. Prevention of type 2 diabetes mellitus by changes in lifestyle among subjects with impaired glucose tolerance. N Engl J Med 2001; 344: 1343-1350.

7. Knowler WC, Barrett-Connor E, Fowler SE, Hamman RF, Lachin JM, Walker EA Nathan DM; Diabetes Prevention Program Research Group. Reduction in the incidence of type 2 diabetes with lifestyle intervention or metformin. N Engl J Med 2002; 346: 393-403.

8. Look AHEAD Research Group. Reduction in Weight and Cardiovascular Disease Risk Factors in Individuals With Type 2 Diabetes: One-Year Results of the Look AHEAD Trial. Diabetes Care. 2007; 30:1374-1383.

9. Dixon JB, O'Brien PE, Playfair J, Chapman L, Schachter LM, Skinner S, Proietto J, Bailey M, Anderson M. Adjustable gastric banding and conventional therapy for type 2 diabetes: a randomized controlled trial. JAMA. 2008;299:316-623.

10. Sjöström L, Review of the key results from the Swedish Obese Subjects (SOS) trial- a prospective controlled intervention study of bariatric surgery. J Intern Med 2013; 273: 219-234.

11. World Health Organizacion. BMI Classification. http://apps. who.int/bmi/index.jsp?introPage=intro_3.html

12. Mason EE, Ito C. Gastric bypass in obesity. Surg Clin North Am. 1967; 47:1345-1351

13. Rubino F, Schauer PR, Kaplan LM, Cummings DE. Metabolic Surgery to Treat Type 2 Diabetes: Clinical Outcomes and Mechanisms of Action. Annu. Rev. Med. 2010. 61:393-411.

14. Buse JB, Caprio S, Cefalu WT, Ceriello A, Del Prato S, Inzucchi SE, McLaughlin S, Phillips GL 2nd, Robertson RP, Rubino F, Kahn R, Kirkman MS. How do we define cure of diabetes? Diabetes Care. 2009; 32:2133-2135.

15. Clinical Guidelines on the Identification, Evaluation, and Treatment of Overweight and Obesity in Adults: The Evidence Report http://www.nhlbi.nih.gov/guidelines/obesity/ob_gdlns.htm 
16. Rubio MA, Martínez C, Vidal O, Larrad A, Salas-Salvado J, Pujol J, Díez I. Moreno B. Documento de consenso sobre cirugía bariátrica. Rev Esp Obes. 2004; 4: 223249.

17. Runkel N. Evidence-based german guidelines for surgery for obesity. Int J Colorectal Dis. 2011; 26:397-404.

18. Rubino F, Kaplan LM, Schauer PR, Cummings DE. The Diabetes Surgery Summit Consensus Conference: recommendations for the evaluation and use of gastrointestinal surgery to treat type 2 diabetes mellitus. Ann Surg. 2010; 251: 399-405.

19. Vetter ML, Cardillo S, Rickels MR, Iqbal N. Narrative Review: Effect of Bariatric Surgery on Type 2 Diabetes Mellitus. Ann Intern Med. 2009; 150:94-103.

20. Dixon JB, le Roux CW, Rubino F, Zimmet P. Bariatric surgery for type 2 diabetes. Lancet. 2012; 379:2300-2311.

21. Soni A, Shukla AP, Rubino F. Interventional Diabetology: The Evolution of Diabetes Care in the XXI Century. Curr Atheroscler Rep. 2012; 14:631-636.

22. Schauer PR, Burguera B, Ikramuddin S, Cottam D, Gourash W, Hamad G, Eid GM, Mattar S, Ramanathan R, Barinas-Mitchel E, Rao RH, Kuller L, Kelley D. Effect of laparoscopic Roux-en $\mathrm{Y}$ gastric bypass on type 2 diabetes mellitus. Annals of Surg. 2003; 238:467-484.

23. Sareli AE, Cantor CR, Williams NN, Korus G, Raper SE, Pien G, Hurley S, Maislin G, Schwab RJ. Obstructive Sleep Apnea in Patients Undergoing Bariatric Surgery. A Tertiary Center Experience. Obes Surg. 2011;21:316-327

24. Jamal M, Wegner R, Heitshusen D, Liao J, Samuel I. Resolution of hyperlipidemia follows surgical weight loss in patients undergoing Roux-en-Y gastric bypass surgery: a 6-year analysis of data. Surgery for Obesity and Related Diseases. 2011; 473-479.

25. Nguyen T, Lau DC. The Obesity Epidemic and Its Impact on Hypertension. Canadian Journal of Cardiology. 201228:326-333.

26. Vincent HK, Ben-David K, Cendan J, Vincent KR, Lamb KM, Stevenson $A$. Effects of bariatric surgery on joint pain: a review of emerging evidence. Surg Obes Relat Dis. 2010; 6:451-460.

27. Romeo S, Maglio C, Burza MA, Pirazzi C, Sjöholm K, Jacobson P, Svensson PA, Peltonen M, Sjöström L, Carlsson LM. Cardiovascular Events After Bariatric Surgery in Obese Subjects With Type 2 Diabetes. Diabetes Care. 2012; 35:2613-2617.

28. Sjöström $L$, Peltonen $M$, Jacobson $P$, Sjöström $C D$, Karason $K$, Wedel H, Ahlin S, Anveden Å, Bengtsson C, Bergmark G, Bouchard C, Carlsson B, Dahlgren S, Karlsson J, Lindroos AK, Lönroth H, Narbro K, Näslund I, Olbers T, Svensson PA, Carlsson LM. Bariatric Surgery and Long-term Cardiovascular Events JAMA. 2012; 307:56-65.

29. Sjöström L, Narbro K, Sjöström CD, Karason K, Larsson B, Wedel H, Lystig T, Sullivan M, Bouchard C, Carlsson B, Bengtsson C, Dahlgren S, Gummesson A, Jacobson P, Karlsson J, Lindroos AK, Lönroth H, Näslund I, Olbers T, Stenlöf K, Torgerson J, Agren G, Carlsson LM; Swedish Obese Subjects Study. Effects of Bariatric Surgery on Mortality in Swedish Obese Subjects, N Engl J Med. 2007; 357:741-752.

30. Sjöström L, Gummesson A, Sjöström CD, Narbro K, Peltonen M, Wedel H, Bengtsson C, Bouchard C, Carlsson B, Dahlgren S, Jacobson P, Karason K, Karlsson J, Larsson B, Lindroos AK, Lönroth H, Näslund I, Olbers T, Stenlöf K, Torgerson J, Carlsson LM; Swedish Obese Subjects Study. Effects of bariatric surgery on cancer incidence in obese patients Lancet Oncol. 2009; 10:653-662.

31. Sjöström $L$, Lindroos $A K$, Peltonen $M$, Torgerson J, Bouchard C, Carlsson B, Dahlgren S, Larsson B, Narbro K, Sjöström CD, Sullivan M, Wedel $\mathrm{H}$; Swedish Obese Subjects Study Scientific Group. Lifestyle, diabetes, and cardiovascular risk factors 10 years after bariatric surgery. N Engl J Med. 2004; 351:2683-2693.

32. Jones KB, Jr. Experience withth eRoux-en-Y gastric bypass, and commentary on current trends. Obes Surg. 2000; 10:183-185.
33. Cummings DE, Overduin J, Foster-Schubert KE. Gastric bypass for obesity: Mechanisms of weight loss and diabetes resolution. J Clin Endocrinol Metab. 2004; 89:2608-2615.

34. Schauer PR, Burguera B, Ikramuddin S, Cottam D, Gourash W, Hamad G, Eid GM, Mattar S, Ramanathan R, Barinas-Mitchel E, Rao RH, Kuller L, Kelley D. Effect of laparoscopic Roux-en $Y$ gastric bypass on type 2 diabetes mellitus. Ann Surg. 2003; 238:467.

35. Sugerman HJ, Wolfe LG, Sica DA, Clore JN. Diabetes and hypertension in severe obesity and effects of gastric bypass-induced weight loss. Ann Surg. 2003; 237:751.

36. Buchwald $\mathrm{H}$, Avidor $\mathrm{Y}$, Braunwald $\mathrm{E}$, Jensen MD, Pories W, Fahrbach K, Schoelles K. Bariatric surgery: A systematic review and meta-analysis. JAMA. 2004;292:1724.

37. Dixon JB, Dixon AF, O'Brien PE. Improvements in insulin sensitivity and cell function (HOMA) with weight loss in the severely obese. Homeostatic model assessment. Diabet Med. 2003; 20:127-134.

38. Sala PC, Torrinhas RS, Heymsfield SB, Waitzberg DL. Type 2 Diabetes Mellitus: A Possible Surgically Reversible Intestinal Dysfunction. Obes Surg. 2012; 22:167-176.

39. Yamaoka K, Tango T. Efficacy of lifestyle education to prevent type 2 diabetes: a meta-analysis of randomized controlled trials. Diabetes Care. 2005; 28:2780-2786.

40. Sjöström L, Review of the key results from the Swedish Obese Subjects (SOS) trial. A prospective controlled intervention study of bariatric surgery. J Intern Med 2013; 273: 219-234.

41. Carlsson LM, Peltonen M, Ahlin S, Anveden Å, Bouchard C, Carlsson B, Jacobson P, Lönroth H, Maglio C, Näslund I, Pirazzi C, Romeo S, Sjöholm K, Sjöström E, Wedel H, Svensson PA, Sjöström L.. Bariatric surgery and prevention of type 2 diabetes in Swedish Obese Subjects. N Engl J Med, 2012; 367: 695-704.

42. Bariatric Surgical and Procedural Interventions in theTreatment of Obese Patients with Type 2 Diabetes A position statement from the IDF Taskforce on Epidemiology and Prevention 2011. En http://www.idf.org/webdata/docs/IDF-Position-StatementBariatric-Surgery. Ultimo ingreso: mayo 2013.

43. Dixon JB, le Roux CW, Rubino F, Zimmet P. Bariatric surgery for type 2 diabetes. Lancet. 2012; 379: 2300-2311.

44. Pories WJ, Dohm LG, Mansfield CJ. Beyond the BMI: the search for better guidelines for bariatric surgery. Obesity. 2010; 18:865-871.

45. Sjöström L. Review of the key results from the Swedish Obese Subjects (SOS) trial. A prospective controlled intervention study of bariatric surgery. J Intern Med 2013; 273:219-234.

46. Livingston $\mathrm{E}$. Inadequacy of $\mathrm{BMI}$ as an Indicator for Bariatric Surgery. JAMA, 2012; 307:99,89.

47. Buchwald H Consensus Conference Panel. Consensus Conference Statement Bariatric surgery for morbid obesity: Health implications for patients, health professionals, and third-party payers Surgery for Obesity and Related Diseases. 2005; 1:371-381.

48. Dixon JB, Zimmet P, Alberti KG, Rubino F. Bariatric surgery: an IDF statement for obese type 2 diabetes. Diabet Med 2011; 28: 628-642.

49. Zimmet P, Alberti KGMM, Rubino F, Dixon JB. IDF's view of bariatric surgery in type 2 diabetes. Lancet 2011; 378: 108-110.

50. Lautz D,Halperin F, Goebel-Fabbri A,Goldfine A. The great debate: medicine or surgery: what is best for the patient with type 2 diabetes? Diabetes Care. 2013; 13:16-24.

51. Arterburn DE, Bogart $A$, Sherwood NE, Sidney $S$, Coleman $K J$, Haneuse S, O'Connor PJ, Theis MK, Campos GM, McCulloch D, Selby J. Multisite study of long -term remission and relapse of type 2 diabetes mellitus following gastric bypass. Obes Surg. 2013; 23:93-102. 
52. Laferrère $B$, Swerdlow $N$, Bawa $B$, Arias $S$, Bose M, Oliván B, Teixeira J, McGinty J, Rother KI. Rise of oxintomodulin in response to oral glucose after gastric bypass surgery in patiens with type 2 diabetes. J Clin Endocrinol Metab. 2010; 95:4072-4076.

53. Strader AD, Vahl TP, Jandacek RJ, Woods SC, D'Alessio DA, Seeley RJ. Weight loss through ileal transposición is accompanied by increased ileal hormone secretion and synthesis in rats. Am J Psysiol Endocrinol Metab. 2005; 288:E447-E453.

54. Rubino F, Marescaux J. Effect of duodenal-yeyunal exclusión in a non-obese animal model of type 2 diabetes: a new perspective for an old diseases. Ann Surgery. 2004; 239:1-11.

55. Rubino F, Forgione A, Cummings DE, Vix M, Gnuli D, Mingrone $\mathrm{G}$, Castagneto M, Marescaux J.The mechanism of diabetes control after gastrointestinal bypass surgery reveals a role of the proximal small intestine in the pathophysiology of type 2 diabetes. Ann Surg. 2006; 244:741-749.

56. Kirchner $H$, Gutierrez JA, Solenberg PJ, Pfluger PT, CzyzykTA, Willency JA, Schürmann A, Joost HG, Jandacek RJ, Hale JE, Heiman ML, Tschöp MH. GOAT links dietary lipids with the endocrine control of energy balance. Nat Med. 2009;15:741-745.

57. Li Z, Zhang HY, Lv LX, Li DF, Dai JX, Sha O, Li WQ, BaiY, Yuan L. Roux-en-Y gastric bypass promotes expression of PDX-1 and regeneration of beta-cell in Goto-Kakizaki rats. World J Gastroenterol 2010; 16:2244-2251.

58. Kohli R, Setchell KD, Kirby M, Myronovych A, Ryan KK, Ibrahim SH, Berger J, Smith K, Toure M, Woods SC, Seeley RJ. A surgical model in male obese rats uncovers protective effects of bile acids post-bariatric surgery. Endocrinology. 2013; 154:2341-2351.
59. Pournaras DJ, Glicksman C, Vincent RP, Kuganolipava S, Alaghband-Zadeh J, Mahon D, Bekker JH, Ghatei MA, Bloom SR, Walters JR, Welbourn R, le Roux CW. The role of bile after Rouxen-Y gastric bypass in promoting weight loss and improving glycaemic control. Endocrinology. 2012; 153:3613-3619.

60. van der Ven N. Psychosocial Group Interventions in Diabetes Care. Diabetes Spectrum. 2003; 16:88-95.

61. Krause N, Herzog AR, Baker E: Providing support to others and well-being in later life. J Gerontol. 1992; 47:300-P311.

62. Heisler M. Overview of Peer Support Models to Improve Diabetes Self-Management and Clinical Outcomes. Diabetes Spectrum. 2007; 20:214-221.

63. Gallant MP. The influence of social support on chronic illness self-management: a review and directions for research. Health Educ Behav. 2003; 30:170-195.

64. Funnell MM The Diabetes Attitudes, Wishes, and Needs (DAWN) Study Clinical Diabetes. 2006; 24:154-155.

65. Kownacki RJ, Shadish WR: Does Alcoholics Anonymous work? The results from a meta-analysis of controlled experiments. Subst Use Misuse. 1999; 34:1897-1916.

66. Life After Bariatric Surgery. University of California. San Francisco. http://www.ucsfhealth.org/education/life_after_bariatric_ surgery/index.html. Último ingreso 02/06/2013. 